\title{
Dynamic modeling of a closed loop jet mill
}

\author{
H.J.C. Gommeren ${ }^{\text {a }}$, D.A. Heitzmann ${ }^{\text {a }}$, H.J.M. Kramer ${ }^{\text {a }}$, \\ K. Heiskanen ${ }^{\mathrm{b}}$, B. Scarlett ${ }^{\mathrm{a}}$ \\ ${ }^{a}$ Delft University of Technology, Delfi, Netherlands \\ ${ }^{\mathrm{b}}$ Helsinki University, Espoo, Finland
}

\begin{abstract}
Although jetmilling is a very energy consuming grinding process it is increasingly used in industry because very fine grinding product with a narrow size distribution is attained without contamination as the milling occurs by inter particle collisions. At Delft University a project has been started to achieve a considerable energy reduction of the milling process. Main feature of the improved grinding system will be an operation control based on in-line particle size measurement using the laser diffraction technique. The pilot plant is equipped with an external classifier for the removal and recirculation of larger particles leaving the jet mill.

Phenomena occurring in the mill are not yet well modeled and thus no real optimization of the grinding process can be achieved. Operation conditions of jet mills are still determined by trial and error.

This paper is to form the basis of a model which allows a dynamic modeling of the behavior of the jet mill/classifier system. The closed loop grinding system has been split in 3 subsystems (zones), two internal to the mill and one external through which the particles pass. Exchange of particles is not described by complicated residence time distribution functions but by "particle size" deprendent probability functions.
\end{abstract}

\section{Introduction}

In a spiral jet mill high pressure air ( $7 \mathrm{bar}$ ) is tangentially injected in the mill chamber creating a rotating flow (see Fig. 1). Solids fed in the mill chamber are transferred to the outer zone where they are hit by jets. Size reduction is caused by mutual collisions. Fine particles are dragged to the central outlet.

In a jet mill optimization for grinding and classification performance can not be achieved simultaneously, because this jet mill is normally operated in such a way that the number of particles larger than the desired product size is minimized. This leads to a highly inefficient grinding process in terms of energy consumption. 


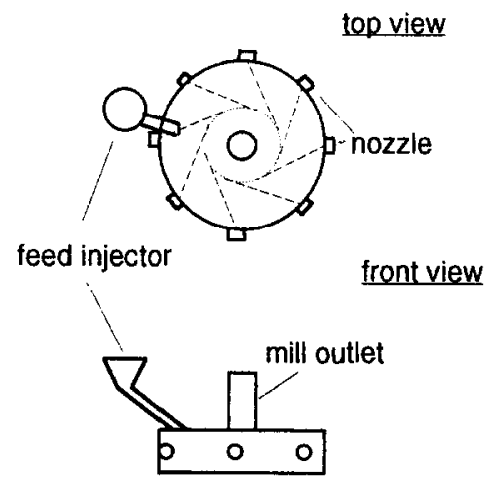

Fig. 1. Spiral jet mill.

For research purpose a grinding pilot plant has been built with an external classifier which recirculates the coarse fraction to the jet mill. Fines are collected as end product.

To predict whether the grinding process is stable and, if so, to improve the transient responses of the PSD by some sort of feed back control, dynamic models are required. This paper deals mainly with the mathematical modeling of the grinding system.

\section{Grinding system and model construction}

Fig. 2 shows a schematic representation of the lay out of the pilot plant. As can be seen, the classical jet mill system has been extended with an external classifier.

The modeling will only consider what is happening inside the defined system boundary. To structurize the theoretical modeling, the closed loop grinding system is divided in subsystems, called zones.

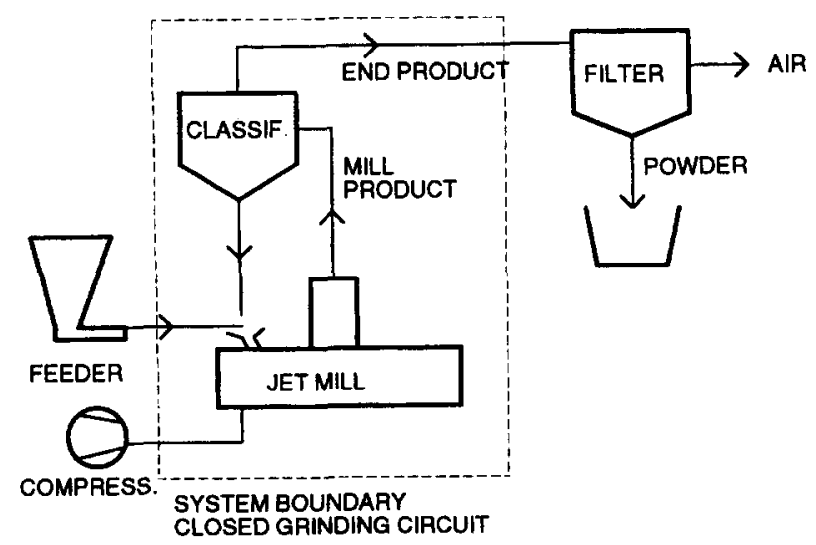

Fig. 2. Schematic picture of the closed loop grinding system. 


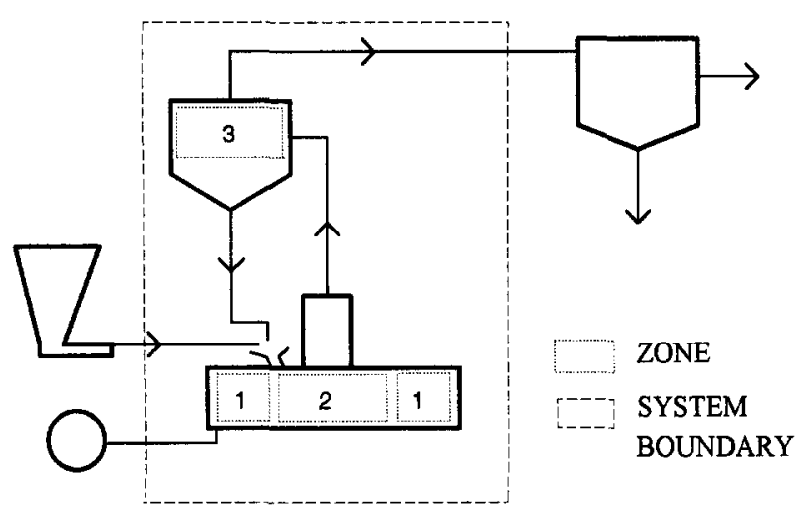

Fig. 3. Subsystems (zones) of the closed loop grinding system.

Inside the mill chamber 2 zones are distinguished (see Fig. 3) called grinding zone (1) and central zone (2). The external classifier is defined as zone 3 . In the open loop configuration (no external classifier), zone 3 is the outlet of the jet mill. Each of those zones has a specific action: zone 1 is the grinding zone, zone 2 and 3 the internal and external classification zones. Both in the jet mill chamber as in the external air classifier solid particles are classified according to their flow behaviour in zones where they are subjected to centrifugal forces and drag forces existing in rotational airflows. Based on equipment geometry and inlet velocity a theoretical expected cutsize in the flow field of a zone can be calculated.

However, the real separation in a vortex flow is influenced by stochastic disturbances due to the turbulence of the air stream, particle wall interactions and particle particle interactions, and can thus not be described by a single cutsize.

Therefore the particle transfer between the different zones of the system is described by size dependent probability functions.

\section{Mathematical formulation}

\subsection{Zone 1}

For the comminution process the concepts of breakage function $\left(B_{i j}\right)$ and selection for breakage $\left(S_{i}\right)$ proposed by Epstein are used (Austin, 1971).

The selectivity $S_{i}\left(\mathrm{~s}^{-1}\right)$ gives the percentage of particles of size $x_{i}$ submitted to too weak forces and therefore sustaining, and is described by the commonly used formula:

$$
S_{i}=\alpha\left(x_{i} / x_{\max }\right)^{\lambda}
$$

The breakage function $B_{i j}(i \geq j)$ predicts how broken particles of a certain size classe $j$ appear in smaller size classes $i$, and is written as:

$$
B_{i, j}=\left[\phi\left(x_{i} / x_{j}\right)^{\gamma}+(1-\phi)\left(x_{i} / x_{j}\right)^{\beta}\right]
$$


where $\alpha, \beta, \gamma, \lambda$ are parameters, and $b_{i, j}=B_{i j}-B_{i+1, j}$ is the density breakage function.

For simulation purposes, the transfer probabilitics between the zones are described by S-shaped curves, e.g. cumulative log-normal distributions. Particles have a probability $P_{12, i}$ to go from zone 1 to zone 2 per unit time dependent on their size $(i)$. In formula:

$$
P_{12, i}=k \cdot\left[1-0.5\left[1+\operatorname{erf}\left(\left(\ln x_{i}-\ln x_{50 \_12}\right) / \sqrt{2} \ln \sigma_{12}\right)\right]\right]
$$

where $\chi_{50_{-} 12}$ is the cut size, $\ln \sigma_{12}$ is the variance of $\ln \left(\chi / \chi_{50 \_12}\right)$, and $k\left(s^{-1}\right)$ is the scale factor.

\subsection{Zone 2}

The transfer of particles to zone 1 is described by $P_{21, i}=k-P_{12, i}$, while transfer to zone 3 is given by an equation similar to (3.3) but with other parameters.

\subsection{Zone 3}

The separation efficiency curve $Y_{\mathrm{cls}, i}$ of the external classifier is described by the same kind of equation as $P_{21, i}$, but $Y_{\mathrm{cls}, i}$ is dimensionless while $P_{21, i}$ is in s ${ }^{-1}$.

\subsection{Population balance}

The population balance for size class $i$ over zone 1 between time $t$ and $t+\Delta t$ is:

$$
\begin{aligned}
m_{1, i}(t+\Delta t)= & m_{1, i}(t)+m_{2, i}(t) P_{21, i} \Delta t+\sum_{j=1}^{i} b_{i, j} S_{j} m_{1, j}(t) \Delta t \\
& -m_{1, i}(t) P_{12, i} \Delta t-S_{i} m_{1, i}(t) \Delta t
\end{aligned}
$$

where

$m_{\mathrm{a}, i}(t)$

$M_{\mathrm{a}}(t)$

$f_{\mathrm{a}, i}(t)$

$m_{2, i}(t) P_{21, i} \Delta t$

$m_{1, i}(t) P_{12, i} \Delta t$

$S_{i} f_{1, i}(t) \Delta t$

$\sum_{j=1}^{i} b_{i, j} S_{j} m_{1, j}(t) \cdot \Delta t$
Mass of size class $i$ at time $t$ in zone

$\mathrm{a}=M_{\mathrm{a}}(t) \cdot f_{\mathrm{a}, i}(t)$

Hold-up in zone a at time $t$

Density size distribution in zone a at time $t$

(\% in class $i$ )

Mass of particles with size $i$ transferring from zone [g]

2 to zone 1

Mass of particles with size $i$ transferring from zone $\quad[\mathrm{g}]$

1 to zone 2

Mass of particles of size $i$ that disappear to lower size classes

Particles of size $i$ that appear from higher

size classes by breakage during $\Delta t$

The total flow of particles leaving the mill at time $t$ is:

$$
\Phi_{\mathrm{mp}}(t)=\sum_{i=1}^{\text {ndim }} m_{2, i}(t) \cdot P_{23, i}(t)
$$


Table 1

Standard values of parameters

\begin{tabular}{|c|c|c|c|}
\hline Selectivity & & $\alpha=4\left[s^{-1}\right]$ & $\lambda=0.5$ \\
\hline Breakage & & $\phi=1$ & $\gamma=1$ \\
\hline Feed rate & & $\Phi_{\mathrm{nf}}=10$ & {$[\mathrm{~g} / \mathrm{s}]$} \\
\hline Probabilities & $\begin{array}{l}P_{12} \\
P_{21} \\
P_{23}\end{array}$ & $\begin{array}{l}\sigma_{12}=2 \\
\sigma_{21}=2 \\
\sigma_{23}=1.4\end{array}$ & $\begin{array}{l}d 50_{12}=50 \mu \\
d 50_{21}=50 \mu \\
d 50_{23}=10 \mu\end{array}$ \\
\hline
\end{tabular}

and the coarse part which is recirculated to the mill is:

$$
\Phi_{\mathrm{rr}}(t+\tau)=\sum_{i=1}^{\mathrm{ndim}} m_{2, i}(t) \cdot P_{23, i}(t) \cdot Y_{\mathrm{cls}, i}
$$

Thus, the population balance over zone 2 becomes:

$$
\begin{aligned}
m_{2, i}(t+\Delta t)= & m_{2, i}(t)+\left\{\Phi_{\mathrm{nf}}(t) \cdot f_{\mathrm{nf}, i}(t)+\Phi_{\mathrm{rr}}(t-\tau) \cdot f_{\mathrm{rr}, i}(t-\tau)\right\} \cdot \Delta t \\
& +m_{1, i}(t) \cdot P_{12, i} \cdot \Delta t-m_{2, i}(t) \cdot\left[P_{21, i}+P_{23, i}\right] \cdot \Delta t
\end{aligned}
$$

where

$\Phi_{\mathrm{nf}}(t), \Phi_{\mathrm{rf}}(t) \quad$ Massflow of new feed (nf), respectively recycled flow (rf) $\quad[\mathrm{g} / \mathrm{s}]$ $f_{\mathrm{nf}, i}(t), f_{\mathrm{rf}, i}(t) \quad$ Normalized population density in new feed, respectively $[-]$ recirculating flow (rf)

\section{Simulation results}

In a jet mill system the breakage parameters are obviously dependent on the hold up and the size distribution inside the mill. The internal classifying action of the mill is probably function of those variables as well.

In order to get a first idea of the model, simulations were done while keeping all those parameters constant in time. Table 1 contains the the standard values used in the

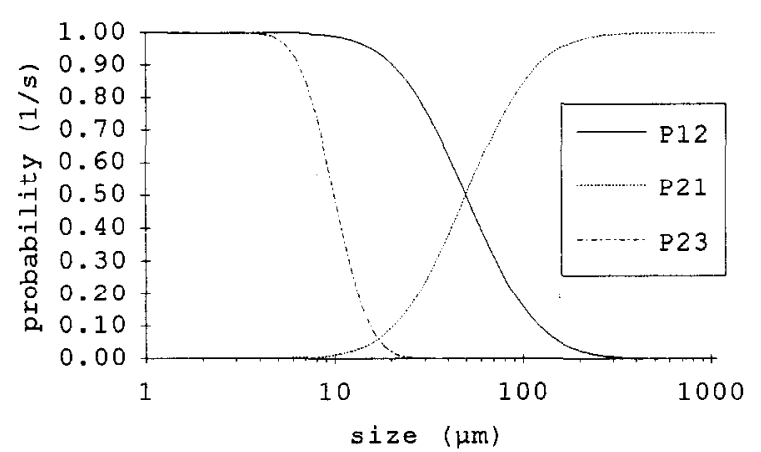

Fig. 4. Transfer probabilitities of particle between zones as a function of their size. 


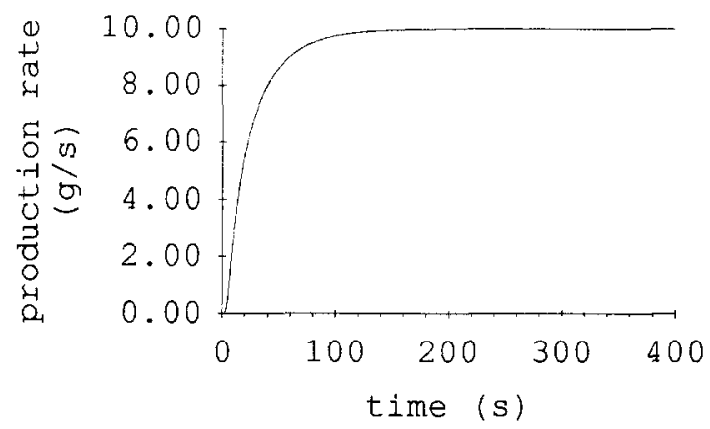

Fig. 5. Production rate.

simulations. Fig. 4 illustrates the different transfer probabilities in the mill when using values of Table 1 .

\subsection{Open loop simulations}

In the open loop configuration of the plant the external classifier is "switched off", so no particles are recirculated to the mill chamber.

Using the standard parameter values (Table 1), Fig. 5 shows the evolution of production rate with time. From this simulations it turns out that the process gets into a steady state condition after about 150 seconds.

This time scale is consistent with some experimental results due to Schäfer and Sommer (1986) who showed that a stationary condition is established after 3 to 4 minutes in their jet mill.

Fig. 6 shows the PSD of the end product in the steady state conditions. The internal classifier function $P_{23}$, which turns out to have the biggest influence on the final size distribution, has been optimised by fitting it with experimental results obtained on an industrial $70 \mathrm{~cm}$ jet mill.

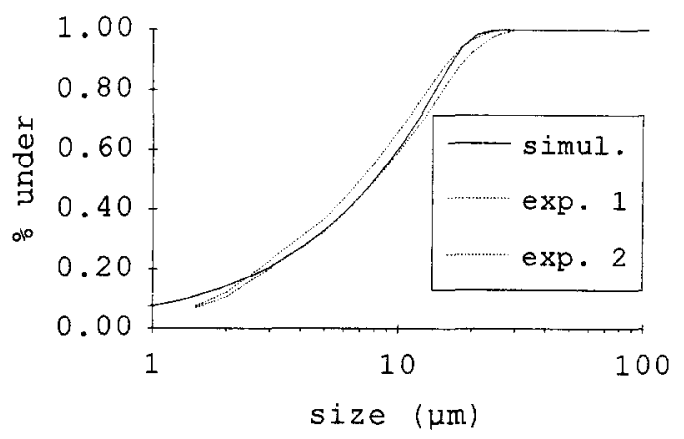

Fig. 6. Milled product size distribution at steady state. 


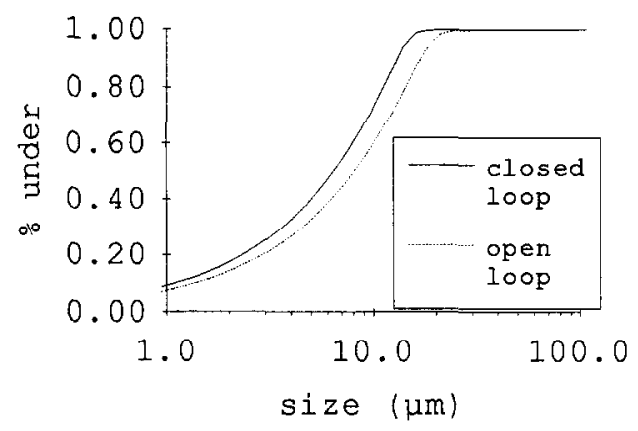

Fig. 7. Size distribution of end product.

\subsection{Closed loop simulations}

The external classifier is now active. Its grade efficiency curve has been chosen equal to that of the internal classifier (see $P_{23}$ ). Under this conditions a recirculating load of $17 \mathrm{~g} / \mathrm{s}$ is obtained at a feed rate of $10 \mathrm{~g} / \mathrm{s}$. Reasonable recirculating loads are 2 to 3 times the product yield (Heiskanen, 1993).

Fig. 7 shows the PSD of the end product for the open loop and closed loop cases. The cumulative PSD is shifted into the direction of the smaller particles, which indicates that finer product is produced at the same feed rate. The $d_{50}$ value is shifted from $8.0 \mu \mathrm{m}$ to $6.3 \mu \mathrm{m}$.

As can be expected in practice and seen in Fig. 8, it takes a longer time to reach the steady state in the closed loop configuration.

The time needed to reach $95 \%$ of the nominal rate is 80 seconds in open loop and 158 seconds in closed loop.

Fig. 9 shows that the total hold up in the jet mill chamber is almost doubled in case the external classifier is used.

In Fig. 10 the influence of different selection for breakage functions is shown (see Eq. 3.2). A higher $\alpha$ means that more particles are selected for breakage per unit time. A more efficient comminution results in a slightly finer end product.

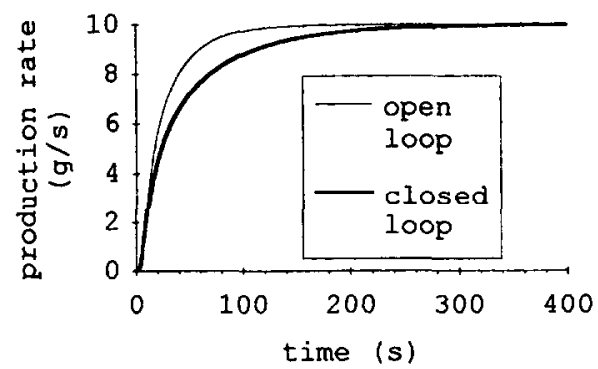

Fig. 8. Production rate as function of time. 


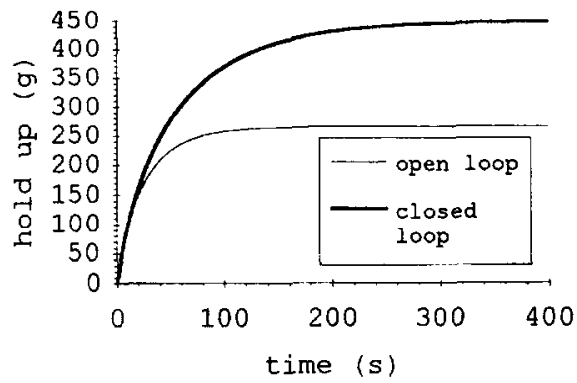

Fig. 9. Total hold up in the jet mill chamber changing in time.

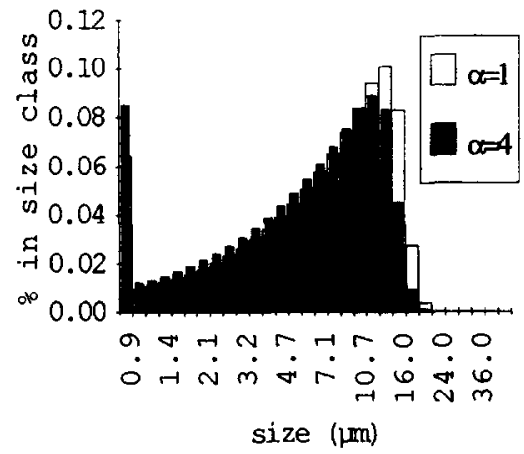

Fig. 10. PSD of end product for two selectivity functions.

\subsection{RTD simulations}

The program has the possibility to simulate a pulse injection of material with a given size and to trace its fragments in the output. This allows the determination of the residence time distribution of this material as is done by radioactive tracer measurements.

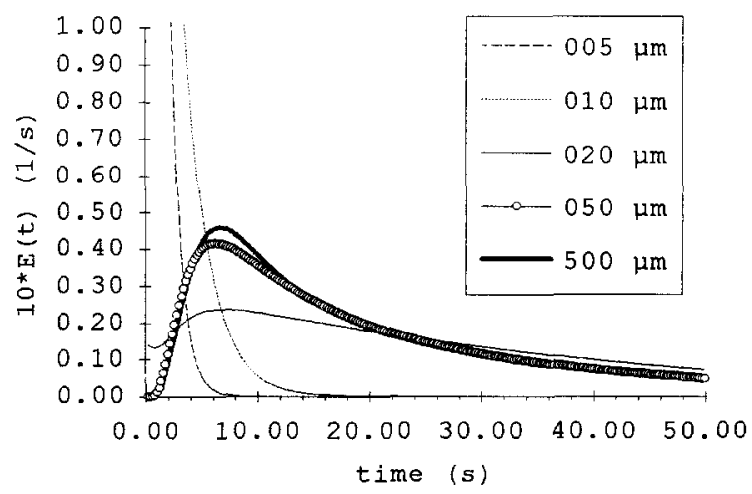

Fig. 11. RTD for different sizes of particles. 


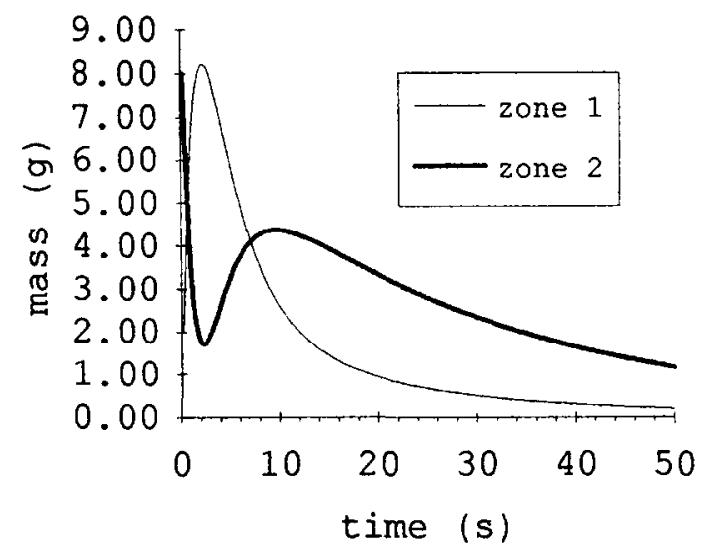

Fig. 12. Evolution of mass in zones 1 and 2 after a pulse injection.

Fig. 11 shows the RTD for different sized particles. The mean residence time for the indicated sizes varies from 4 seconds for the smallest to 80 seconds for the largest particles.

Fig. 12 shows how particles are moving through the mill after a pulse tracer injection of $10 \mathrm{~g}$ of 500 micron particles in the central zone (2). During the first few seconds the amount of tracer in zone 2 decreases as particles are transfered to zone 1 where they are ground. After a while, the tracer fragments reappear in zone 2 before they leave the mill.

The influence of the breakage selectivity $(\alpha=4 ; 2 ; 1)$ on the RTD of particles with a size of 50 micron is investigated. As $\alpha$ decreases, the mean residence time $\tau$ increases from 131 to 216 and 366 seconds. For a less efficient breakage (lower $\alpha$ ), $\tau$ is larger, as expected.

\section{Discussion and conclusion}

In the open loop configuration the internal classification function $P_{23}$ is mainly resporisible for the location and shape of the PSD-curve. Therefore this function is optimised to fit the experimental data (see Fig. 6). The breakage parameters $\alpha$ has only a slight influence on this curve (Fig. 8).

The: simulations show that an external classifier has three main effects: producing a finer end product, increasing the time to reach the steady state, and rising the mill hold up weight. The latter can have insidious effects on the breakage and separation efficiencies and future simulations should take this into account. This simulations have proved to give rather realistic results, although no parameter adjustments have been performed to take into account variations in hold ups and size distributions in the mill during the non steady state phase.

The main advantage of this model over the use of RTDs is that only three probability curves $P_{i j}$ are necded to describe the stochastic flow behavior of the particles in the mill, instead of $\mathrm{n}$ RTD curves for a full description by the classical models ( $n=$ number of size classes). 
The other advantage is that the addition of an external classifier is done by simply adding the separation efficiency curve.

\section{References}

Austin, L.G., 1971. Introduction to the mathematical description of grinding as a rate process. Powder Technol., 5: 1-17.

Heiskanen, K., 1993. Particle Classification. Chapman and Hall, London, 321 pp.

Schäfer, W. and Sommer, K., 1986. Influences of dispersion and convection during grinding on the solid distribution in a spiral jet mill. In: 1st World Congress Particle Technology, Nümberg, Part II, Comminution, pp. 325-341. 